\title{
Wirksamkeit und Durchführbarkeit von Graphästhesie-Training bei Patienten mit chronisch unspezifischen Rückenschmerzen
}

\author{
Randomisierte kontrollierte Pilotstudie \\ Effectiveness and Feasibility of Graphaesthesia Training in Patients \\ with Non-specific Chronic Low Back Pain
}

\author{
Randomised Controlled Pilot Study
}

\author{
Autoren \\ Katharina van Baal, Fiona Schwarz, Katja Ehrenbrusthoff, Christian Grüneberg
}

Institut

Hochschule für Gesundheit, Bochum

Schlüsselwörter

chronische Rückenschmerzen, sensomotorischer Kortex, physiotherapeutische Behandlungsverfahren, randomisierte kontrollierte Studie, Graphästhesie

Key words

chronic low back pain, sensorimotor cortex, physiotherapy modalities, randomised controlled trial, graphaesthesia

eingereicht 20.04.2017

akzeptiert 10.11.2017

Bibliografie

DOI https://doi.org/10.1055/a-0600-5121

physioscience 2018; 14: 57-68

(c) Georg Thieme Verlag KG, Stuttgart · New York ISSN 1860-3092

\section{Korrespondenzadresse}

Katharina van Baal, PT

MSc Evidence-based Health Care, Gesundheitscampus 6-8, 44801 Bochum katharina.vanbaal@hs-gesundheit.de

\section{ZUSAMMENFASSUNG}

Hintergrund Chronisch unspezifischer Rückenschmerz (CURS) stellt ein erhebliches Gesundheitsproblem dar. Aktuelle Studien weisen darauf hin, dass kortikale Veränderungen bei den betroffenen Patienten ein beitragender Faktor für die Persistenz von Schmerzen sein können. Bei anderen Patienten mit chronischen Schmerzen und nachgewiesenen kortikalen Veränderungen konnte die Wirksamkeit sensorischer Trainingsansätze gezeigt werden. Unklar ist, ob diese Ansätze auch bei Patienten mit CURS wirksam sind.

Ziel Das Ziel dieser Pilotstudie war es, die Größenordnung möglicher Effekte des Graphästhesie-Trainings einzuschätzen und dessen Durchführbarkeit zu überprüfen.
Methoden 9 Probanden wurden randomisiert in 2 Gruppen eingeteilt. In der 3-wöchigen Interventionsphase erhielt die Kontrollgruppe physiotherapeutische „Usual Care“, während die Interventionsgruppe zusätzlich 2-mal wöchentlich ein 20-minütiges Graphästhesie-Training absolvierte.

Ergebnisse Im Vergleich der beiden Messzeitpunkte innerhalb sowie zwischen den Gruppen zum Messzeitpunkt 2 fanden sich keine signifikanten Unterschiede. Deskriptiv zeigte sich die Tendenz einer verbesserten Graphästhesie-Fehlerrate und Zweipunktdiskriminationsschwelle sowie von Schmerzintensität, -interferenz und körperlicher Funktion in der Interventionsgruppe.

Schlussfolgerung Das Graphästhesie-Training ist im klinischen physiotherapeutischen Kontext durchführbar und führt nicht zu unerwünschten Nebenwirkungen. Bezüglich der Wirksamkeit bestehen lediglich Tendenzen. Aufgrund des Pilotcharakters der Arbeit sind die Ergebnisse mit Vorsicht zu interpretieren. Es bedarf Folgestudien mit größeren Studienpopulationen zu kombinierten sensomotorischen Trainingsprogrammen bei CURS, insbesondere erweitert um den Aspekt einer vorangehenden Klassifizierung der Patienten in Subgruppen.

\section{ABSTRACT}

Background Non-specific chronic low back pain (NSCLBP) is a major health problem. Current studies indicate that cortical alterations may be a contributing factor to persistent pain in affected patients. In other patients with chronic pain and identified cortical alterations, sensory training approaches showed initial effectiveness. It is unclear whether these approaches are also effective in patients with NSCLBP.

Objective The aim of this pilot study was to assess the degree of possible treatment effects of a graphaesthesia training and to investigate its feasibility.

Method Nine patients were randomised into two groups. During the three-week intervention period the control group received usual physiotherapy care whilst the intervention group performed an additional 20 minutes graphaesthesia training twice a week. 
Results The comparison of the two measurement points within the groups as well as between the groups at measurement point two showed no significant differences. Descriptive data revealed a tendency of improved graphaesthesia error rates and two-point discrimination thresholds as well as pain severity, pain interference and physical function in the intervention group.

Conclusions In the clinical physiotherapeutic context graphaestesia training is feasible and does not lead to any unwan- ted adverse effects. Regarding the effectiveness there exist only tendencies. Due to the pilot character of the study, the results should be interpreted with caution. More research studies with larger sample sizes regarding combined sensorimotor exercise programmes for NSCLBP are necessary, especially extended by a preceding classification of patients into subgroups.

\section{Einleitung}

Rückenschmerzen sind eines der meist verbreiteten Krankheitsbilder in Deutschland. Dies zeigt unter anderem die Stichprävalenz von Rückenschmerzen, die ohne Angabe des Schweregrads „am heutigen Tag“ in einer Umfrage des Robert Koch Instituts in verschiedenen Regionen Deutschlands bei $32-49 \%$ liegt [1].

Aufgrund der erheblichen Ausgaben für das Gesundheitssystem gehört insbesondere chronischer Rückenschmerz zu den kostenintensivsten Erkrankungen in Deutschland. Die durch chronische Rückenschmerzen verursachten volkswirtschaftlichen Schäden lagen 2010 mit 17,5 Milliarden Euro an der Spitze im Vergleich von chronischen Erkrankungen bei Arbeitnehmern [2]. Im Jahr 2013 wurden annähernd die Hälfte aller physiotherapeutischen Heilmittelverordnungen in Deutschland aufgrund von Erkrankungen der Wirbelsäule ausgestellt [3].

Insgesamt liegt der Anteil unspezifischer Rückenschmerzen bei ca. $85 \%$ und macht damit den größten Teil der Patienten mit Rückenschmerzen aus [4, 5]. Rückenschmerzen sind unspezifisch, wenn es keine Hinweise auf eine spezifische Ursache im Sinne einer Diagnose gibt. Die Diagnose eines spezifischen Rückenschmerzes wird gestellt, wenn ein nachweisbarer Zusammenhang zwischen den Rückenschmerzen und einer definierten Ursache besteht [6]. Die Tatsache, dass bislang wenig über die Ursachen des großen Anteils an chronischen unspezifischen Rückenschmerzen (CURS) bekannt ist, könnte eine Ursache für die begrenzte Wirksamkeit aktueller Therapien sein [7].

Aufgrund der erheblichen Relevanz von CURS für das deutsche Gesundheitssystem liegt eine Vielzahl an Therapieangeboten vor. Die Mehrheit der untersuchten Therapien, wie z. B. Kinesio-Taping, Interferenzstromtherapie, therapeutischer Ultraschall und Kältetherapie werden als nicht wirksam beschrieben und nicht für Patienten mit CURS empfohlen [6, 8]. Die Nationale Versorgungsleitlinie Nicht-spezifischer Kreuzschmerz [6] empfiehlt für die Behandlung aktuell aktive Bewegungstherapie kombiniert mit edukativen Maßnahmen, Rehabilitationssport, Entspannungsverfahren und kognitive Verhaltenstherapie.

Sensorische Trainingsansätze finden in den Empfehlungen der Nationalen Versorgungsleitlinie bisher keine Berücksichtigung. Der Fokus liegt in einer Vielzahl der zugrundeliegenden Studien auf strukturell-funktionellen Behandlungsweisen, obwohl Forschungsergebnisse eine geringere Bedeutsamkeit struktureller Veränderungen bei CURS nahelegen [9-11]. Aktuelle Forschungsergebnisse lassen die Vermutung zu, dass persistierende
Schmerzen bei diesen Patienten ihre Ursache unter anderem in der kortikalen Reorganisation haben könnten [7 - 11]. Die Messung der taktilen Wahrnehmungsschärfe wird als das klinische Korrelat zur kortikalen Reorganisation angesehen und liefert somit Informationen über das Ausmaß der sensorischen (Dys)funktion der Patienten [12].

Bei Patienten mit CURS zeigte sich, dass eine kortikale Reorganisation im Bereich des primären somatosensorischen Kortex (S1) stattfindet [11, 13, 14], die als eine Veränderung der Repräsentation des betroffenen Körperareals im primären somatosensorischen Kortex definiert werden kann [10, 15 - 17]. Die Veränderungen auf S1 scheinen bei der Entwicklung und Aufrechterhaltung von chronischem Schmerz eine Rolle zu spielen $[10,18]$. Obwohl zunehmend von einer Relevanz der kortikalen Veränderungen bei CURS ausgegangen wird, gilt es weiter zu untersuchen, ob diese wirklich eine Ursache, Wirkung oder Folge der Symptomatik sein können $[10,11]$.

Ähnliche Veränderungen wie bei Patienten mit CURS finden sich auch bei Patienten mit Amputationen [19] oder Complex Regional Pain Syndrome [15-17]. „Cortical Remapping“ als eine neuronale Reorganisation im Gehirn ist demnach ein bekannter Faktor bei chronischen Schmerzsyndromen. Ein klinischer Hinweis auf die kortikale Reorganisation ist bei Patienten mit Complex Regional Pain Syndrome oder Phantomschmerz nach Amputationen eine erhöhte Schwelle bei der Zweipunktdiskrimination $[15,19]$. Forschungsergebnisse legen nahe, dass sensorisches Diskriminationstraining die Schmerzen der Patienten mit Complex Regional Pain Syndrome lindert und ihre taktile Wahrnehmungsschärfe verbessert [20]. Durch ein sensorisches Training soll sich die Veränderung auf dem somatosensorischen Kortex zurückbilden lassen, und diese Rückbildung einen Einfluss auf die Schmerzwahrnehmung der Patienten haben [15]. Allerdings gibt es noch keine Studie, die einen Bezug zur körperlichen Funktionsfähigkeit und Lebensqualität von Patienten mit chronischen Schmerzerkrankungen herstellt.

Auch Patienten mit CURS haben Defizite der taktilen Wahrnehmungsschärfe [21]. Dies bezieht sich auf eine signifikante Vergrößerung der Zweipunktdiskriminationsschwelle gegenüber gesunden Probanden [12, 21, 22]. Die Messung der taktilen Wahrnehmungsschärfe erfolgt mithilfe der Zweipunktdiskrimination. Es soll der Mindestabstand in Millimetern ermittelt werden, bei dem es Patienten möglich ist, zwei von einem Punkt an ihrem Rücken voneinander zu unterscheiden [23]. 
Zusätzlich zu einer Vergrößerung der Zweipunktdiskriminationsschwelle fanden Moseley et al. [22] bei Patienten mit CURS eine Übereinstimmung der erhöhten Zweipunktdiskriminationsschwelle mit dem Schmerzgebiet der Patienten und des Areals einer eingeschränkten Körperwahrnehmung mittels Körperschemazeichnungen. Die Zweipunktdiskriminationsschwelle unterschied sich an anderen Stellen des Rückens nicht von den Werten der Kontrollgruppe, was ein weiterer Hinweis auf eine herabgesetzte taktile Wahrnehmungsschärfe und ein verändertes Körperschema im Schmerzgebiet bei dieser Patientengruppe ist [22].

Forschergruppen stellten die Hypothese auf, dass Graphästhesie-Training eine geeignete Therapie zur Steigerung der taktilen Wahrnehmungsschärfe bei Patienten mit CURS sein kann [11, 21]. Die Graphästhesie (griech. Graph: „schreiben“, Ästhesie: „Wahrnehmen/Empfinden“) wird mithilfe des entsprechenden gleichnamigen Tests erhoben. Dieser wird definiert als „sensorischer Test, bei dem der Patient Zahlen erkennen muss, welche der Therapeut auf z. B. Handrücken, Arme, Rumpf und Fußrücken schreibt“ [24]. Die Aufgabe erfordert nicht nur eine gute taktile Wahrnehmungsfähigkeit, sondern zusätzlich eine kortikale Konstruktion des gezeichneten Symbols und stellt somit eine höhere Anforderung dar [25]. In aktuellen Forschungsarbeiten zeigten Patienten mit CURS eine erhöhte Fehlerrate bei der Graphästhesie im Vergleich zu gesunden Probanden [21].

Die Auffälligkeiten der Patienten mit CURS bei beiden genannten Testverfahren bekräftigen die Vermutung, dass eine kortikale Reorganisation mit Auswirkungen auf messbare Veränderungen der taktilen Wahrnehmung stattfindet [21]. Hieraus ergibt sich der Therapieansatz der vorliegenden Pilotstudie.

In dieser Pilotstudie wurden primär erste Hinweise auf die Größenordnung möglicher Effekte eines Graphästhesie-Trainings auf die körperliche Funktionsfähigkeit, die psychische Komponente der gesundheitsbezogenen Lebensqualität sowie den Schmerz bei Patienten mit CURS in einem randomisierten kontrollierten Studiendesgin generiert. Sekundär wurden die Auswirkungen auf die sensorischen Testverfahren untersucht und die Durchführbarkeit der Intervention in der unmittelbaren Versorgungsrealität der ambulanten Heilmittelerbringung überprüft.

\section{Methode}

\section{Studiendesign}

Die Ethikkommission des Deutschen Verbandes für Physiotherapie (ZVK) e. V. genehmigte die randomisierte kontrollierte Pilotstudie und sie wurde im deutschen Register klinischer Studien mit der Registrierungsnummer DRKS00007116 registriert.

Die Interventionsgruppe wurde mit physiotherapeutischer „Usual Care“ und mit 2-mal wöchentlichem zusätzlichen Graphästhesie-Training behandelt. Die Kontrollgruppe erhielt ausschließlich physiotherapeutische „Usual Care“. Dabei wurden im Prä-post-Vergleich die genannten primären Outcome-Parameter betrachtet. Sekundär fand die Untersuchung der sensorischen Testverfahren sowie der Durchführbarkeit und unerwünschten Nebenwirkungen der Intervention statt. Außerdem erfolgte mithilfe der Ergebnisse eine Powerkalkulation für die Berechnung der Stichprobengröße für Folgestudien.

\section{Probanden}

Die Probanden wurden aus einer Kooperationseinrichtung der Hochschule für Gesundheit rekrutiert. In einer 1. Phase wurden alle Therapeuten der Einrichtung über Inhalt und Ablauf der Studie sowie die Ein- und Ausschlusskriterien informiert. Aufgabe der Therapeuten war es, in einer Vorlaufphase von etwa 2 Wochen allen in Frage kommenden Probanden die Informationen auszuhändigen und um die Erlaubnis der Weitergabe der Kontaktdaten für einen Telefonanruf durch die Studienleiter zu bitten. Diese führten telefonisch ein anamnestisches Screening durch und luden geeignete Patienten ein ( $\triangleright$ Abb. 1).

Die Studienteilnehmer mussten mindestens 18 Jahre alt sein und unter CURS mit oder ohne Ausstrahlung in die Extremitäten von mindestens 3 Monaten Dauer leiden. Gemäß der genannten Definition unspezifischer Rückenschmerzen durfte keine spezifische Diagnose vorliegen, die einen direkten Zusammenhang mit der aktuellen Symptomatik aufwies. Gemäß aktuellen Empfehlungen $[5,26]$ wurden die Patienten anhand der Einteilung in eine diagnostische Triage nach Waddell gescreent [27, 28]. Demnach lassen sich die spezifischen Rückenschmerzen in spinale Pathologien mit ernsthaften Ursachen und radikuläre Syndrome aufteilen. Probanden mit derartigen spezifischen Rückenschmerzen wurden ausgeschlossen. Außerdem mussten die Teilnehmer zum Zeitpunkt der Erhebung in konventioneller physiotherapeutischer Behandlung sein und über gute deutsche Sprach- und Schriftkenntnisse verfügen. Personen mit klinischen oder anamnestischen Hinweisen auf schwerwiegende Erkrankungen, Hautveränderungen oder Narben im Bereich der LWS, erheblichen kognitiven Einschränkungen, Schwangerschaft oder ärztlicher Behandlung bezogen auf den Rückenschmerz im Erhebungszeitraum waren ausgeschlossen.

Alle Probanden erhielten vor Studienbeginn eine Teilnehmerinformation und unterschrieben eine Einverständniserklärung.

Zu Beginn der Rekrutierung wurden insgesamt 22 potenzielle Probanden über die Studie informiert. Davon waren 11 Probanden bereit, an der Studie teilzunehmen. Jeweils 1 Teilnehmer aus der Interventions- und Kontrollgruppe musste während der Interventionsphase aus persönlichen Gründen der Probanden die Studie beenden ( $\boldsymbol{A}$ Abb. $\mathbf{1})$. Daraus ergab sich eine abschließende Probandenzahl von $\mathrm{n}=9$ mit 4 Teilnehmern in der Interventionsund 5 in der Kontrollgruppe.

Die Werte der beiden Probanden, die nicht bis zum Studienende teilnahmen, wurden nicht in die Auswertung eingeschlossen. Demnach fand keine Intention-to-treat-Analyse statt.

\section{Randomisierung und Verblindung}

Die Zuteilung zu beiden Gruppen erfolgte mithilfe einer Software (DatInfRandList Version 1.2), die die Probanden im Verhältnis 1:1 den beiden Gruppen zuordnete. Dabei wurden die Ziffern 0 und 1 für Kontroll- und Interventionsgruppe in zufälliger Reihenfolge 11-mal aneinandergereiht. Die Probanden wurden in der Abfolge der ausgegebenen Randomisierung der Gruppe 0 oder 1 zugeordnet, indem die Umschläge mit den Daten der Probanden 


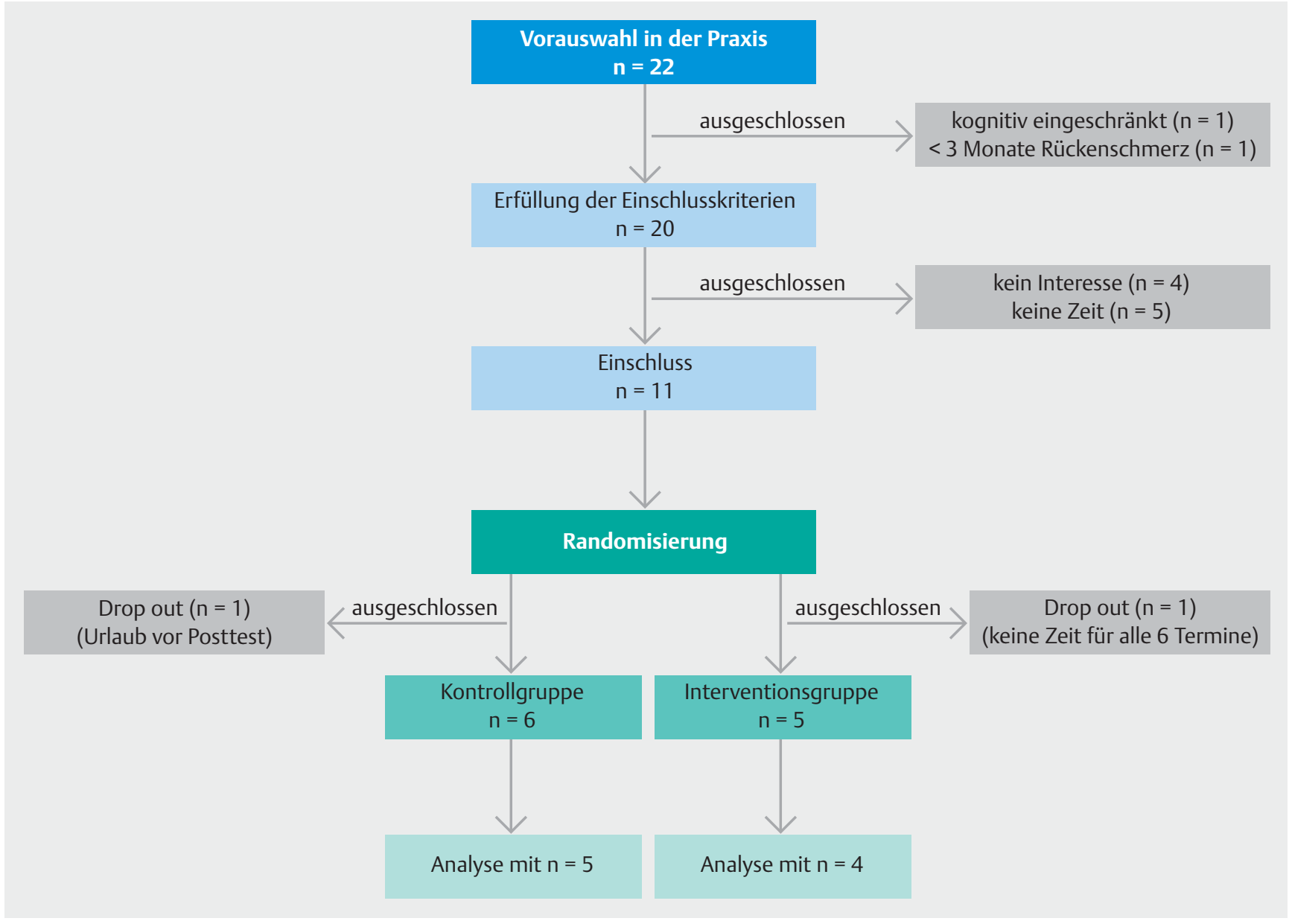

- Abb. 1 Flow-Chart der Teilnehmerrekrutierung. (Quelle: K. van Baal; graf. Umsetzung: Thieme Gruppe).

ohne sichtbare Kennzeichnung in der Reihenfolge ihrer Erhebung im Prätest aufeinandergelegt wurden. Die Behandlerin der Interventionsgruppe führte die Randomisierung durch, da deren Verblindung während der Interventionsphase ohnehin nicht möglich gewesen wäre. Die Untersucherin war gegenüber der Gruppenzuteilung verblindet. Eine Verblindung der Probanden war aufgrund der Art der Intervention nicht möglich.

\section{Assessments}

Vor Beginn der Interventionen wurden zum Messzeitpunkt T1 alle Outcome-Parameter erhoben und zusätzlich die folgenden demografischen Basisdaten aufgenommen: Alter, Größe, Gewicht, Gesamtschmerzdauer, Einnahme von Medikamenten, derzeitige physiotherapeutische sowie ärztliche Behandlungen, Arbeitsstatus, Krankschreibung, vorhandene neurologische Erkrankungen, Schwangerschaft und Narben oder Hauterkrankungen am unteren Rücken.

Beim Messzeitpunkt T2 nach Beendigung des Interventionszeitraums wurden alle Outcome-Parameter erneut erhoben. Aufgrund der zeitlich begrenzten Ressourcen der Studienleiterinnen war kein weiterer Messzeitpunkt vor Interventionsbeginn zwecks einer Baseline-Phase möglich.

\section{Primäre Outcome-Parameter}

Primäre Outcomes waren die mittels Selbstbeurteilungsfragebögen erhobenen patientenrelevanten Parameter: gesundheitsbezogene Lebensqualität (Short-Form 36 Gesundheitsfragebogen, SF-36), körperliche Funktionsfähigkeit (Roland-Morris Disability Questionnaire, RMDQ) sowie Schmerzintensität und -interferenz (Brief Pain Inventory, BPI).

\section{Lebensqualität}

Lebensqualität gilt als ein bedeutender Endpunkt für die Beurteilung von Patienten mit CURS. Das für die Erhebung bei dieser Patientengruppe empfohlene Testverfahren ist der SF-36 [29]. Der Fragebogen umfasst in 8 Unterskalen aufgeteilte 36 Items, die verschiedenen Dimensionen der Lebensqualität entsprechen [30]. In der vorliegenden Arbeit wurden die 4 Einzelitems der Summenskala zur psychischen Gesundheit „Vitalität“, „soziale Funktionsfähigkeit“, „emotionale Rollenfunktion“ und „psychische Gesundheit“ sowie deren Summenskala betrachtet [30]. Die Einschätzungen für die einzelnen Items werden summiert und in einen Wert zwischen 0 und 100 umgerechnet, mit 100 als bestmöglichem Wert. Der Normwert zur psychischen Summenskala liegt in einer gesunden Population bei 50, wobei Abweichungen nach unten eine verringerte und nach oben eine erhöhte Lebensqualität anzeigen [30, 31]. 6 
von 8 mit dem SF-36 gemessene Unterskalen der gesundheitsbezogenen Lebensqualität zeigen eine sehr gute interne Konsistenz [32].

\section{Körperliche Funktionsfähigkeit}

Der RMDQ ist ein Selbstbeurteilungsfragebogen für Patienten mit Rückenschmerzen, der die Funktionseinschränkung/körperliche Behinderung misst [33]. Diese ist ein relevanter Endpunkt, weil die betroffenen Personen erhebliche Einschränkungen beschreiben [34, 35]. Der RMDQ besteht aus 24 als Aussagen formulierten Items. Der maximale Summenscore beträgt 24, was der höchstmöglichen Beeinträchtigung entspricht [34]. Die deutsche Version des Fragebogens wurde an einer Gruppe von Patienten mit Rückenschmerzen validiert und zeigt gute Werte für die Test-Retest-Reliabilität sowie die interne Konsistenz [35, 36].

\section{Schmerz}

Für die Erfassung von Schmerz kam die Kurzfassung der deutschen Version des BPI zum Einsatz. Er gilt in der klinischen Praxis und Forschung als Standard und ist in deutscher Sprache validiert [37]. Der Fragebogen besteht aus 15 Fragen und deckt die Dimensionen Schmerzintensität (4 Items) und -interferenz (7 Items) ab, während die übrigen Items als optional betrachtet werden [38]. Für die Schmerzintensität und -interferenz liegen die Werte zwischen 0 und 10, die einer Numeric Rating Scale entsprechen und bei der 10 den größtmöglichen Schmerz darstellt [39]. Die Validität und Reliabilität der deutschen Version des BPI zeigt anhand einer Gruppe von allgemeinen Patienten mit Schmerzen und Krebserkrankungen mit der Originalversion vergleichbare gute Werte [37].

\section{Sekundäre Outcome-Parameter}

Sekundäre Outcome-Parameter waren die sensorischen Testverfahren und die Durchführbarkeit der Intervention. Für beide sensorischen Testungen wurden die Probanden in Bauchlage auf der Behandlungsliege mit freiem unteren Rücken gelagert. Zunächst zeichnete die Untersucherin mit einem wasserlöslichen Stift den Punkt ein, den die Probanden als üblicherweise schmerzhafte Stelle angaben. Dieser Punkt bildete die Mitte für die Einzeichnung des $25 \mathrm{~cm}^{2}$ großen Gebietes für den Graphästhesie-Test. Der mittig eingezeichnete Schmerzpunkt bildete das Zentrum für die Messung der Zweipunktdiskrimination.

Bei Probanden mit bilateralen Schmerzen wurde mittels Münzwurf zufällig entschieden, auf welcher Seite die Testung stattfinden sollte. Die genaue Einzeichnung bestimmte die Untersucherin zufällig, befand sich aber immer im Schmerzgebiet. Die Testung beider Messverfahren geschah anhand von zuvor standardisierten Durchführungsprotokollen.

Vor der Durchführung an den Studienteilnehmern übte die Untersucherin das Verfahren an freiwilligen gesunden Probanden in 2 Sitzungen von jeweils 2 Stunden Dauer.

\section{Graphästhesie-Test}

Für den Graphästhesie-Test wurde den Probanden eine standardisierte Anweisung vorgelesen. Das zuvor abgegrenzte Gebiet von $25 \mathrm{~cm}^{2}$ diente als Maß für die Größe der Buchstaben. Der mit dem Gegenstand auf die Haut ausgeübte Druck war so stark, dass sich beim Einzeichnen gerade eine leichte Weißfärbung der Haut zeigte [21].

Die Studienleiterinnen verwendeten sowohl für die Intervention als auch für das Testverfahren eine Stricknadel mit einem Durchmesser von ca. $3 \mathrm{~mm}$, was in etwa einer breiten Kugelschreiberspitze entspricht. Vor Beginn der Testung wurden alle Buchstaben einmal mit Ansage der Untersucherin gezeichnet. Die tatsächliche Testung bzw. Intervention bestand danach aus 60 Buchstaben in randomisierter Reihenfolge in 3 Sets mit jeweils 20 Buchstaben [21]. Analog zur Graphästhesie-Intervention fand zwischen 2 Buchstaben eine Pause von etwa 5 Sekunden sowie zwischen 2 Sets eine längere Pause von etwa 3 Minuten statt [20]. Die Probanden sollten sich in jedem Fall für einen Buchstaben entscheiden, auch wenn sie sich nicht sicher waren. Die Untersucherin nahm im Rahmen des Testverfahrens keine Korrekturen vor.

Auf einem vorbereiteten Testprotokoll wurde nach jedem Buchstaben die Richtigkeit der Angabe notiert und ein Fehler mit 1 Punkt kodiert. Aus dem Gesamtergebnis errechnete sich auf diese Weise aus den getesteten Buchstaben eine Fehlerrate zwischen 0 und 60 [21].

\section{Zweipunktdiskrimination}

Die Messung der Zweipunktdiskriminationsschwelle orientierte sich am Standardprotokoll von Moberg [23]. Vor Beginn der Messung wurde den Probanden eine standardisierte Anweisung zur Testdurchführung vorgelesen. Sie sollten, „eins“ sagen, wenn sie einen Berührungspunkt spürten und „zwei“ wenn sie 2 Punkte wahrnahmen. Auch bei Unsicherheit sollten sie sich für „eins“ oder „zwei“ entscheiden.

Die Zweipunktdiskriminationsschwelle wurde in horizontaler Ausrichtung mittels eines Aesthesiometers von Baseline gemessen. Diese ist definiert als die geringste Distanz zwischen den beiden Berührungspunkten, bei der die Probanden sicher 2 von einem Berührungspunkt unterscheiden konnten [12]. Die Angaben wurden als sicher gewertet, wenn die Probanden 3-mal in Folge Zweifachreize bei der aufsteigenden bzw. Einfachreize bei der absteigenden Messung unterschieden hatten. Als Schwelle galt der Abstand, bei dem die Probanden bei der aufsteigenden Messung zuerst 2 Punkte erkannten sowie bei der absteigenden Messung der Wert, bei dem sie zuletzt 2 Punkte erkannten.

Die Testung der Zweipunktdiskrimination bestand aus insgesamt 4 Messungen, je 2 in aufsteigender und absteigender Reihenfolge. Bei der aufsteigenden Messung wurde ab $1 \mathrm{~cm}$ und bei der absteigenden ab $10 \mathrm{~cm}$ begonnen, wobei bei jedem Schritt um $5 \mathrm{~mm}$ verringert bzw. erhöht wurde $[12,23]$. Um geschätzte Angaben der Probanden in Bezug auf das Muster der Stimuli auszuschließen, wendete die Untersucherin in zufälligen Abständen sogenannte „Catch Trials“ an, bei denen sie nur einen Punkt berührte [20, 23]. Aus den 4 ermittelten Schwellen wurde ein Mittelwert errechnet, der der Auswertung zugrunde lag.

\section{Durchführbarkeit}

Die Behandlerin der Interventionsgruppe, die zugleich eine der Studienleiterinnen war, beurteilte die Durchführbarkeit der Intervention auf der Basis ihrer eigenen Erfahrungen. Nebenwirkungen 
seitens der Probanden erhob sie ab der 2. Behandlung vor jedem Behandlungstermin mithilfe einer standardisierten Eingangsfrage.

\section{Interventionen}

Die Kontrollgruppe erhielt eine physiotherapeutische Behandlung im Sinne von „Usual Care“, die die angestellten Physiotherapeuten in der ambulanten Praxis im normalen Ablauf weiterführten. Dies implizierte, dass unterschiedliche Therapeuten die Probanden behandelten, was dem normalen Ablauf in der Praxis entsprach. Da die Art der „Usual Care“ maßgeblich von den behandelnden Therapeuten und den jeweiligen Patienten abhing, kann sie nicht präzise beschrieben werden. In der Regel handelte es sich jedoch um eine Kombination aus aktiven und passiven Maßnahmen, wobei die passiven Elemente überwogen. Die entsprechenden Maßnahmen wurden befundentsprechend an die Bedürfnisse der Patienten angepasst. Die „Usual Care“ enthielt jedoch keine sensorischen Trainingsansätze, die dem Graphästhesie-Training ähnelten.

Die Interventionsgruppe erhielt über die gesamte 3-wöchige Interventionsdauer zusätzlich ein Graphästhesie-Training. In jeder Woche fanden 2 Behandlungseinheiten von 20 Minuten Dauer entsprechend des Standard-Rezeptes für Physiotherapie in Deutschland statt. Eine Physiotherapeutin, die weder an den Messungen, noch an der Durchführung der „Usual Care“ beider Gruppen beteiligt war, führte die Therapie durch. Die Therapie fand ausschließlich als Einzeltherapie und in den Räumen einer Physiotherapiepraxis häufig vor oder nach der physiotherapeutischen „Usual Care“ statt.

Das Graphästhesie-Training erfolgte entsprechend des Testverfahrens mithilfe einer Stricknadel. Die Probanden befanden sich dabei in Bauchlage mit einem Kissen unter dem Becken und einer Rolle unter den Sprunggelenken. Analog zum Testverfahren wurden 3 Sets mit jeweils 20 Buchstaben auf einem zuvor abgegrenzten Gebiet von ca. $25 \mathrm{~cm}^{2}$ um den Schmerzbereich gezeichnet. Im Unterschied zum Graphästhesie-Test korrigierte die Therapeutin hierbei mündlich falsche Angaben der Probanden und wiederholte die Zeichnung einmal, um den Lernprozess über die Dauer der Interventionsphase hinweg zu unterstützen.

\section{Datenanalyse}

Die Datenanalyse erfolgte mithilfe von SPSS Version 22. Zur Darstellung der demografischen Daten und des klinischen Status der Probanden dienten deskriptive Statistiken. Zur Überprüfung der Outcome-Parameter kamen Tests für Unterschiedshypothesen zur Anwendung. Aufgrund der kleinen Stichprobengröße wurden Unterschiede zwischen und innerhalb der Gruppen mit nicht parametrischen Testverfahren berechnet.

Gruppenvergleiche zwischen Kontroll- und Interventionsgruppe zu T1 und T2 wurden mit dem Mann-Whitney-U-Test vorgenommen. Zur Überprüfung der Unterschiede zwischen den Zeitpunkten T1 und T2 innerhalb der beiden Gruppen diente der Wilcoxon-Vorzeichen-Rang Test für verbundene Stichproben.

Bei allen statistischen Testverfahren wurde die Signifikanzschwelle auf $p=0,05$ festgelegt. Zur Überprüfung der weiteren Machbarkeit für mögliche Folgestudien erfolgte zudem eine Powerkalkulation mithilfe der Software G*Power [40].
- Tab. 1 Basisdaten der Studienpopulation und Vergleich beider Gruppen zum 1. Messzeitpunkt (T1).

\begin{tabular}{|l|c|l|l|}
\hline Kriterium & $\begin{array}{l}\text { IG (n=4) } \\
\text { MW (SD) }\end{array}$ & $\begin{array}{l}\text { KG (n=5) } \\
\text { MW (SD) }\end{array}$ & $\begin{array}{l}\text { p-Wert T1 } \\
\text { (MWU) }\end{array}$ \\
\hline Alter (Jahre) & $56(6,23)$ & $50(15,47)$ & 0,41 \\
\hline Gewicht (kg) & $76(28,08)$ & $79(20,38)$ & 0,91 \\
\hline $\begin{array}{l}\text { Größe (cm) } \\
\text { Body Mass }\end{array}$ & $172(18,67)$ & $166(11,08)$ & 1 \\
\hline $\begin{array}{l}\text { Index } \\
\text { Schmerzdauer } \\
\text { (Jahre) }\end{array}$ & $12,5(13,08)$ & $12,6(10,97)$ & 1 \\
\hline $\begin{array}{l}\text { IG = Interventionsgruppe; KG = Kontrollgruppe; MW = Mittelwert; } \\
\text { MWU = Mann-Whitney-UTest; SD = Standardabweichung. }\end{array}$ & 0,41 \\
\hline
\end{tabular}

\section{Ergebnisse}

Im folgenden Abschnitt wird über die Intra- und Intergruppenvergleiche zu den verschiedenen Zeitpunkten die Größenordnung möglicher Effekte der Interventionen eingeschätzt. Zusätzlich werden die erfassten Daten zu Nebenwirkungen der Graphästhesie-Intervention beschrieben, um die Durchführbarkeit des Trainings zu beurteilen.

\section{Beschreibung der Stichprobe}

Die gesamte Stichprobe $(n=9)$ umfasste 7 Frauen und 2 Männer. In der Interventionsgruppe waren 3 Frauen und 1 Mann, in der Kontrollgruppe 4 Frauen und 1 Mann vertreten. Die übrigen Basisdaten der Probanden sind in $>$ Tab. 1, die Ausprägung der primären Outcome-Parameter zum Zeitpunkt T1 > Tab. 2 dargestellt.

\section{Vergleich der Gruppen zum Zeitpunkt T1}

Aus den Daten ging hervor, dass Kontroll- und Interventionsgruppe zum Zeitpunkt T1 keine signifikanten Unterschiede bezüglich aller Parameter aufwiesen ( $\checkmark$ Tab. 1, 2). Bezüglich des Merkmals Geschlecht unterschieden sich die beiden Gruppen ebenfalls nicht signifikant (Chi-Quadrat Test: $x^{2}=2,778, p=0,096$ ).

\section{Vergleiche innerhalb der Gruppen (T1-T2)}

Sowohl Kontroll- als auch Interventionsgruppe wiesen keine signifikanten Unterschiede für alle Outcomes im Vergleich der Zeitpunkte T1 und T2 auf ( $\triangleright$ Tab. 3 ).

\section{Vergleich beider Gruppen zum Zeitpunkt T2}

Zwischen den beiden Gruppen ergaben sich bezüglich aller Outcome-Parameter zum Zeitpunkt T2 keine signifikanten Unterschiede ( $\triangleright$ Tab. 2).

\section{Durchführbarkeit und Nebenwirkungen}

Keiner der Probanden beschrieb gegenüber der Behandlerin unangenehme Nebenwirkungen der Behandlung. Außerdem 
- Tab. 2 Mittelwerte der Outcome-Parameter zu beiden Messzeitpunkten (T1 und T2) und Signifikanzwerte im Gruppenvergleich.

\begin{tabular}{|c|c|c|c|c|c|c|}
\hline Kriterium & $\begin{array}{l}\text { IG T1 } \\
\text { MW (SD) }\end{array}$ & $\begin{array}{l}\text { KG T1 } \\
\text { MW (SD) }\end{array}$ & KG-IG T1 (p) & $\begin{array}{l}\text { IG T2 } \\
\text { MW (SD) }\end{array}$ & $\begin{array}{l}\text { KG T2 } \\
\text { MW (SD) }\end{array}$ & KG-IG T2 (p) \\
\hline BPI Pain Severity & $3,86(2,74)$ & $4,85(3,52)$ & 1,0 & $3,13(1,94)$ & $4,1(3,09)$ & 0,56 \\
\hline BPI Pain Interference & $2,46(2,66)$ & $2,57(1,96)$ & 0,91 & $1,29(1,37)$ & $3,8(2,83)$ & 0,56 \\
\hline RMDQ & $6,5(8,27)$ & $5,4(2,30)$ & 0,91 & $6,0(7,79)$ & $5,8(3,56)$ & 0,56 \\
\hline Graphästhesie-Fehlerrate & $24,0(13,59)$ & $19,6(8,26)$ & 0,56 & $14,25(7,59)$ & $17,8(7,33)$ & 0,56 \\
\hline Zweipunktschwelle (mm) & $62,0(9,92)$ & $52,0(13,39)$ & 0,19 & $51,0(4,45)$ & $54,0(14,64)$ & 0,56 \\
\hline Psychische Summenskala & $46,77(12,85)$ & $46,25(9,17)$ & 0,91 & $55,59(8,61)$ & $42,51(10,1)$ & 0,11 \\
\hline Subskalenwert PSYC & $69,0(25,59)$ & $61,6(18,89)$ & 0,73 & $83,0(15,45)$ & $57,80(16,74)$ & 0,06 \\
\hline Subskalenwert EMRO & $58,33(50)$ & $66,67(47,14)$ & 0,91 & $83,33(33,33)$ & $66,67(47,14)$ & 0,73 \\
\hline Subskalenwert SOFU & $65,63(11,97)$ & $80,0(22,71)$ & 0,41 & $84,38(18,75)$ & $75(29,32)$ & 0,73 \\
\hline Subskalenwert VITA & $50,0(29,72)$ & $49,0(17,82)$ & 0,73 & $63,75(22,87)$ & $45(13,23)$ & 0,19 \\
\hline
\end{tabular}

$\mathrm{BPI}=$ Brief Pain Inventory; EMRO = emotionale Rollenfunktion; IG = Interventionsgruppe; $\mathrm{KG}$ = Kontrollgruppe; $\mathrm{MW}=$ Mittelwert; $\mathrm{PSYCH}=$ psychisches Wohlbefinden; RMDQ = Roland-Morris Disability Questionnaire; SD = Standardabweichung; SOFU = soziale Funktionsfähigkeit; T1 = Messzeitpunkt 1; $\mathrm{T} 2$ = Messzeitpunkt 2; VITA = Vitalität.

- Tab. 3 Signifikanzwerte für die Vergleiche innerhalb der Gruppen.

\begin{tabular}{|l|l|l|}
\hline Outcome & IG T1-T2 (p) & KG T1-T2 (p) \\
\hline BPI Pain Severity & 0,72 & 0,07 \\
\hline BPI Pain Interference & 0,14 & 0,23 \\
\hline RMDQ & 0,16 & 0,79 \\
\hline Grafästhesie-Fehlerrate & 0,07 & 0,23 \\
\hline Zweipunktschwelle & 0,07 & 0,68 \\
\hline psychische Summenskala & 0,07 & 0,23 \\
\hline Subskalenwert PSYC & 0,07 & 0,42 \\
\hline Subskalenwert EMRO & 0,18 & 1,0 \\
\hline Subskalenwert SOFU & 0,06 & 0,16 \\
\hline Subskalenwert VITA & 0,14 & 0,47 \\
\hline
\end{tabular}

$\mathrm{BPI}=$ Brief Pain Inventory; EMRO = emotionale Rollenfunktion; IG = Interventionsgruppe; $\mathrm{KG}=$ Kontrollgruppe; $\mathrm{MW}=$ Mittelwert; PSYCH = psychisches Wohlbefinden; RMDQ = Roland-Morris Disability Questionnaire; SD = Standardabweichung; SOFU = soziale Funktionsfähigkeit; T1 = Messzeitpunkt 1; T2 = Messzeitpunkt 2; VITA = Vitalität.

berichtete kein Proband von der Aufnahme neuer Behandlungsformen während der Interventionsphase, die die Ergebnisse hätten verändern können. Bis auf das Ausscheiden zweier Probanden aus persönlichen Gründen beendeten alle die Behandlungsphase. In Bezug auf die Machbarkeit der Intervention berichtete die Behandlerin über keine Probleme bei der Durchführung der Intervention.

Demnach kann die Graphästhesie-Intervention gemäß den Ergebnissen dieser Forschungsarbeit als durchführbar gelten.

\section{Fallzahlkalkulation}

Zur Berechnung der Stichprobengröße für Folgestudien diente die Software $G^{*}$ Power [40] für Fallzahlkalkulationen mit Alpha = 0,05 und entsprechend der Konvention Power $=0,8$. Um signifikante Unterschiede zwischen zwei Gruppen zu finden, wären demnach insgesamt $n=54$ Probanden notwendig.

\section{Zusammenfassendes Ergebnis}

Statistisch konnte insgesamt für keinen der untersuchten Outcome-Parameter eine signifikante Veränderung festgestellt werden. Allerdings zeigte die Darstellung der Ergebnisse auf deskriptiver Ebene Tendenzen für Unterschiede zwischen den beiden Gruppen. Die Interventionsgruppe wies eine Verbesserung der sensorischen Testverfahren auf, während die Kontrollgruppe im Vergleich von T1 zu T2 kaum Veränderung zeigte ( $\vee$ Abb. 2).

Außerdem zeigte die deskriptive Darstellung bei den Werten für die Schmerzintensität ( $\triangleright$ Abb. 3 ) und beim Schmerzinterferenz-Score ( $\triangleright \mathbf{A b b} \mathbf{4} \mathbf{4})$ leichte Verschlechterungen in der Kontrollgruppe. Beide Variablen verbesserten sich im Gegensatz dazu bei der Interventionsgruppe in geringem Maße ( $\mathbf{A} \mathbf{b b} \mathbf{3} \mathbf{3}, \mathbf{4})$.

Die Punktzahl des RMDQ zeigte deskriptiv ebenfalls bei der Interventionsgruppe eine deutlichere Verbesserung als bei der Kontrollgruppe ( $\triangleright$ Abb.5).

Die Durchführbarkeit der Intervention ist gegeben, da diese problemlos anwendbar war und zu keinen Nebenwirkungen bei den Probanden führte.

Die notwendige Stichprobengröße für Folgestudien beläuft sich auf insgesamt $n=54$. 


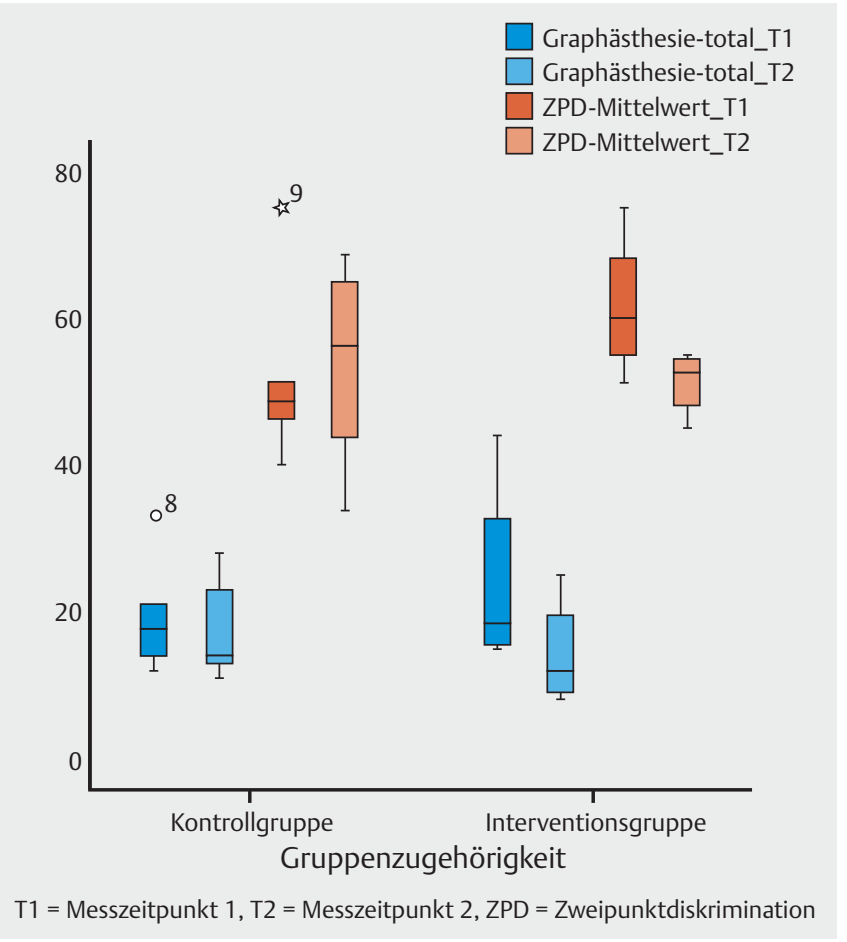

- Abb. 2 Gruppenvergleich der Mittelwerte der sensorischen Testverfahren Prä- und Post-Intervention. (Quelle: K. van Baal; graf. Umsetzung: Thieme Gruppe).

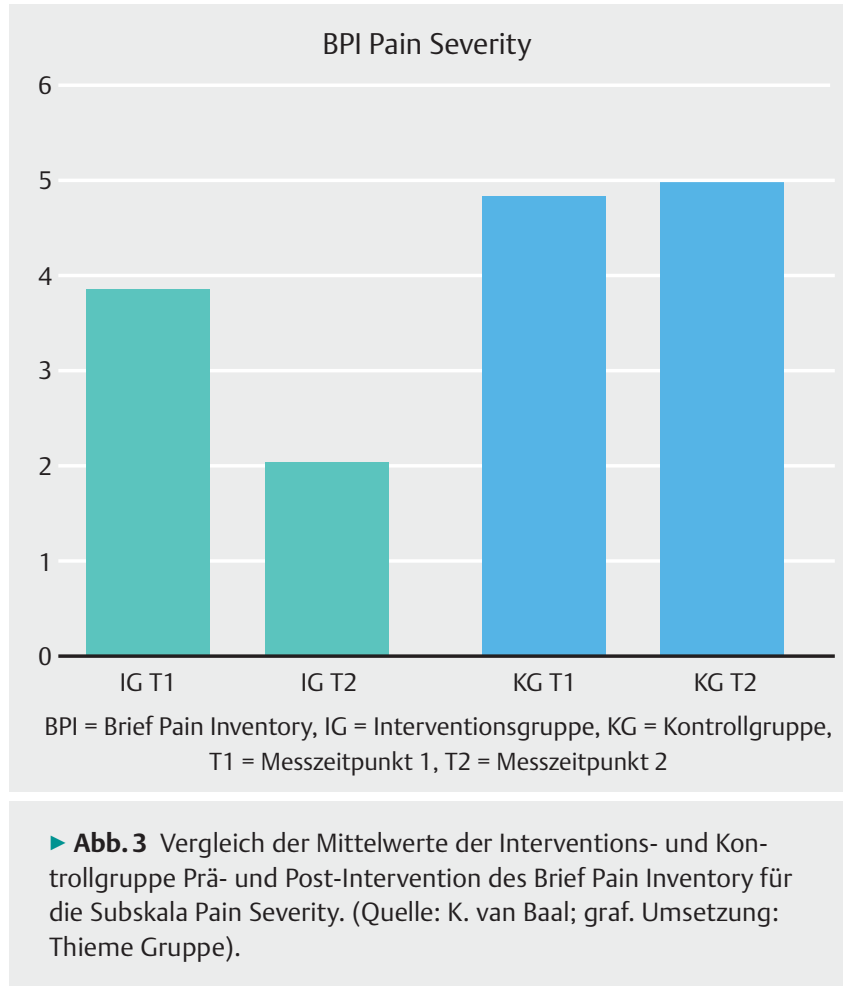

\section{Diskussion}

Das Ziel dieser Pilotstudie war es, erste Daten zur Größenordnung möglicher Effekte eines Graphästhesie-Trainings in Kombination

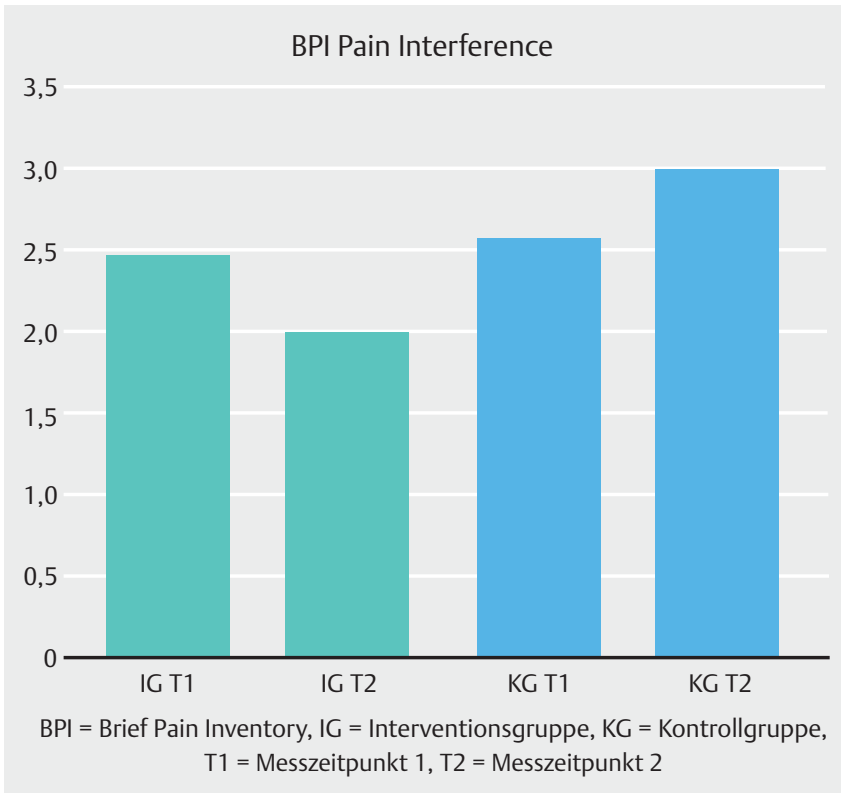

- Abb. 4 Vergleich der Mittelwerte der Interventions- und Kontrollgruppe Prä- und Post-Intervention des Brief Pain Inventory für die Subskala Pain Interference. (Quelle: K. van Baal; graf. Umsetzung: Thieme Gruppe).

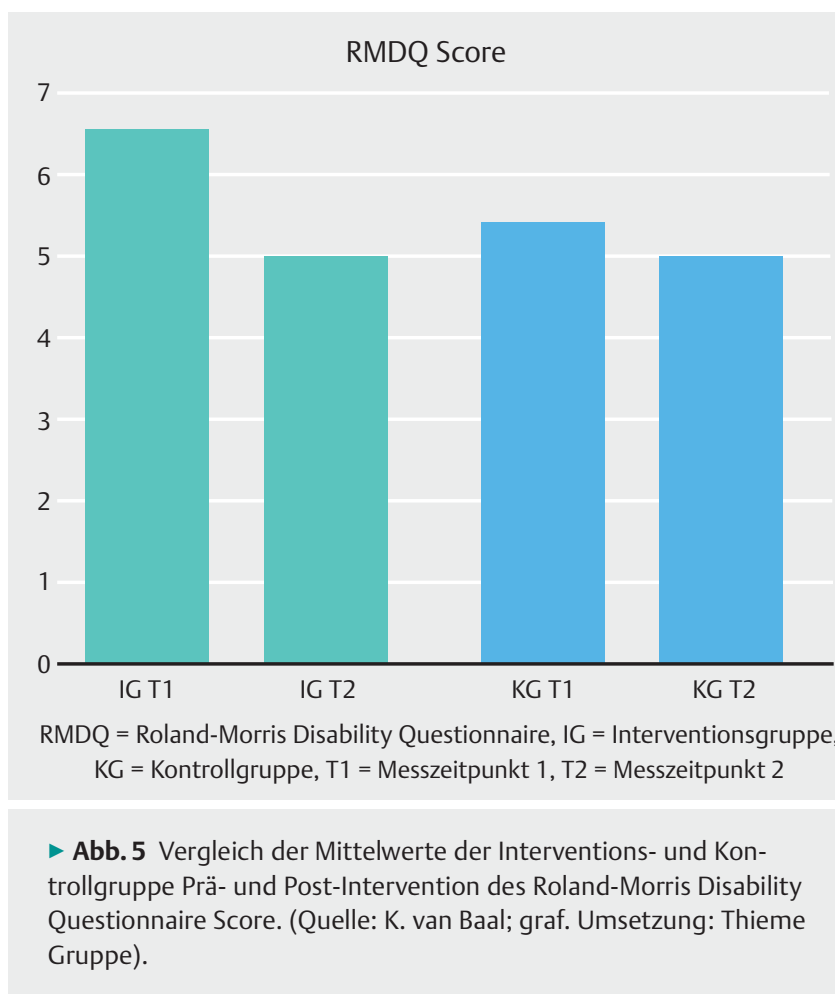

mit „Usual Care“ bei Patienten mit CURS im Vergleich zur alleinigen physiotherapeutischen „Usual Care“ zu sammeln. Die Daten dienten zur Berechnung der Stichprobengröße für weitere Studien. Zudem wurde betrachtet, inwiefern die Intervention durchführbar ist und ob unerwünschte Nebenwirkungen auftreten. 


\section{Hauptergebnisse}

Hauptergebnis dieser Arbeit ist, dass keine signifikanten Unterschiede in allen betrachteten Outcome-Parametern zwischen Kontroll- und Interventionsgruppe beobachtet werden konnten. Ein wichtiges Ergebnis ist jedoch auch, dass die Intervention durchführbar ist und keine unerwünschten Nebenwirkungen auslöst. Die ermittelte Stichprobengröße für Folgestudien liegt bei $n=54$.

\section{Vergleich der Ergebnisse mit anderen Studien}

Die Resultate stehen im Kontrast zu vorherigen Studienergebnissen. Eine Fallserie mit 3 Probanden mit CURS untersuchte die Effekte eines taktilen Wahrnehmungstrainings und zeigte klinisch relevante Verbesserungen bezüglich Schmerz und Funktion [41]. Die ungleichen Ergebnisse könnten darin begründet sein, dass sich das Diskriminationstraining der beiden Studien unterschied. Neben einem Graphästhesie-Training wurden noch andere sensomotorische Trainingsformen angewendet [41], während die vorliegende Pilotstudie keinerlei motorische Komponenten einbezog. Außerdem führten Wand et al. [41] das Training über einen Zeitraum von mindestens 10 Wochen durch, was die Behandlungsdauer von 3 Wochen in dieser Arbeit deutlich übersteigt. Zusätzlich bezogen Wand et al. [41] im Gegensatz zur vorliegenden Arbeit die Komponente der Schmerzedukation ein. Dies könnte die Veränderung der Outcome-Parameter erheblich beeinflusst haben. Durch die andere Interventionsart bei Wand et al. [41] ist nicht auszuschließen, dass das gemessene Konstrukt ein anderes als in dieser Pilotstudie ist.

Wälti et al. [42] untersuchten die Wirksamkeit eines multimodalen Behandlungsprogramms bei einer bestimmten Gruppe von Patienten mit CURS. Die Intervention beinhaltete Edukationsaspekte, sensorisches Training und motorische Kontrollübungen mit zusätzlichen Heimübungen über einen Zeitraum von 8-12 Wochen und zeigte eine hinreichende Durchführbarkeit des Behandlungsprogramms. Dies stimmt mit den Ergebnissen der vorliegenden Arbeit überein. Zusätzlich fanden die Autoren eine signifikante Schmerzreduktion in der Interventionsgruppe als Kurzzeiteffekt im Vergleich zur Kontrollgruppe [42]. Wälti et al. [42] maßen die körperliche Funktion mittels RMDQ, die analog zur vorliegenden Arbeit keine signifikanten Veränderungen zeigte. Die unterschiedlichen Ergebnisse bezüglich einer Veränderung des Schmerzes könnten in der um Aspekte der motorischen Kontrolle und der Schmerzedukation erweiterten Intervention begründet sein.

Andere Studien kommen jedoch zu ähnlichen Ergebnissen wie die vorliegende Arbeit. In einer Pilotstudie im randomisierten, kontrollierten Design überprüften Ryan et al. [43] die Auswirkung von taktilem Diskriminationstraining mittels Graphästhesie. Sie fanden keinen Hinweis darauf, dass das Graphästhesie-Training einen positiven Einfluss auf die Schmerzen und die Funktionsfähigkeit hatte [43]. Es ergaben sich auch keine statistisch signifikanten Unterschiede zwischen beiden Gruppen [43]. Allerdings stellten sie tendenziell eine Verbesserung der Placebo-Gruppe fest [43]. Auch im Vergleich zu dieser Arbeit bestehen methodische Unterschiede. Ryan et al. [43] verglichen mit einer Placebo-Gruppe, die eine rein taktile Stimulation ohne Diskriminationskomponente erhielt. In der Interventionsgruppe führten sie taktiles Diskrimina- tionstraining (Lokalisation und Qualität des Stimulus sowie Graphästhesie) durch und alle Teilnehmer beider Gruppen erhielten zusätzlich physiotherapeutische „Usual Care“. In beiden Gruppen fanden jedoch lediglich 3 Behandlungen durch einen Physiotherapeuten statt, während tägliche Eigenübungen analog zu den jeweiligen Interventionen mit einem nicht professionellen Partner aus dem Umfeld durchgeführt werden sollten. Das Heimprogramm stellte eine große Barriere für eine Vielzahl der Probanden dar, weil es nicht alleine durchgeführt werden konnte [43]. Aus diesem Grund verzichtete die vorliegende Arbeit auf ein Heimübungsprogramm und wich in diesem Aspekt vom Studiendesign bei Ryan et al. [43] ab. Im Gegensatz zu Ryan et al. [43] fand die vorliegenden Arbeit Tendenzen für eine Verbesserung der Interventionsgruppe bezüglich der sensorischen Testfahren sowie der Schmerzinterferenz und des RMDQ-Scores. Die Aussagekraft dieser Tendenzen ist gering, da sich keine statistisch oder klinisch relevanten Veränderungen zeigten.

Insgesamt kamen vorherige Forschungsarbeiten zu sehr unterschiedlichen Ergebnissen. Hieraus ergibt sich gleichzeitig der Bedarf, die Wirksamkeit sensorischer Trainingsprogramme bei Menschen mit CURS weiter zu untersuchen.

\section{Limitationen}

Eine bedeutende Limitation dieser Arbeit ist die dem Pilotcharakter geschuldete Stichprobengröße von $n=9$. Dies schränkt die Aussagekraft der Ergebnisse in Bezug auf die Einschätzung der Größenordnung möglicher Effekte erheblich ein und lässt keine Generalisierung der Ergebnisse zu.

Zusätzlich ist infrage zu stellen, ob eine Gesamtzahl von 6 Interventionen von jeweils 20 Minuten Dauer ausreicht, um Veränderungen bei den patientenrelevanten Outcomes zu bewirken. Andere Arbeiten, die positive Effekte zeigen konnten [41, 42], wendeten die Behandlungsprogramme über einen deutlich längeren Zeitraum an.

Eine weitere Limitation könnte die Ungewissheit über die Art der physiotherapeutischen „Usual Care“ sein, die alle Probanden beider Gruppen erhielten. Da unterschiedliche Therapeuten die Probanden behandelten, kann nicht von einer einheitlichen Therapie ausgegangen werden. Um in Folgestudien verfälschende Aspekte in der Therapie der Kontrollgruppe auszuschließen, sollte ein Manual für die behandelnden Therapeuten verbindliche Vorgaben zur Vereinheitlichung der „Usual Care“ liefern. Ein entscheidender Faktor ist außerdem, dass die Kontrollgruppe keine Placebo-Behandlung zum Ausgleich der Therapiezeit bekam. Die Interventionsgruppe könnte sich demnach tendenziell verbessert haben, weil ihre Teilnehmer mehr Aufmerksamkeit im Sinne eines vermehrten Therapeutenkontaktes erhielten.

Die fehlende Baseline-Phase stellt eine zusätzliche Limitation dar, da aufgrund dessen die Vergleichbarkeit der beiden Gruppen bezüglich der gewählten Outcome-Parameter nicht nachweisbar ist.

Als Begründung für alle genannten Aspekte ist anzuführen, dass die begrenzten zeitlichen und personellen Ressourcen im Rahmen einer Bachelor-Arbeit kombiniert mit der schwierigen Rekrutierung von Probanden keine einheitlicheren Einschlusskriterien erlaubten. 
Bezüglich der Auswahl der Intervention ist infrage zu stellen, ob sich mithilfe des Graphästhesie-Trainings allein die taktile Diskriminationsfähigkeit abbilden lässt und ob diese somit durch die vorliegende Trainingsform verbessert werden kann. Der jetzige Forschungsstand ermöglicht keinen zweifelsfreien Rückschluss darauf, welches Konstrukt bei der Graphästhesie gemessen bzw. behandelt wird. Es stellt sich die Frage, ob die Intervention in zukünftigen Studien erweitert und beispielsweise in ein kombiniertes sensomotorisches Trainingsprogramm gebettet werden muss. Die Auswahl der Intervention in der vorliegenden Studie ist jedoch mit der limitierten verfügbaren Zeit für eine Behandlung im klinischen Alltag in Deutschland zu begründen. Die 20-minütige Intervention orientiert sich demnach an den Gegebenheiten der Versorgungspraxis.

Unklar ist, ob sich die Messung der Zweipunktdiskrimination mit Sicherheit dazu eignet, die Effekte des Graphästhesie-Trainings abzubilden. Der Mangel an verfügbaren, standardisierten und reliablen Messverfahren erklärt aber diese Auswahl. Außerdem ist die Aussagekraft der Zweipunktdiskrimination und des Graphästhesie-Tests als Messverfahren infrage zu stellen, da keine Angaben bzw. im Falle der Zweipunktdiskrimination sehr unterschiedliche Werte zu den Standardfehlern und zu einer Schwelle zur klinischen Relevanz vorliegen [44-47].

Der Vergleich mit anderen Forschungsarbeiten ergab, dass multimodale sensomotorische Trainingsprogramme Effekte zeigen. Insofern sollten Folgestudien kein ausschließlich sensorisches Training untersuchen, sondern zusätzlich Aspekte eines motorischen Trainings sowie der Schmerzedukation mit einbeziehen.

Die Studienpopulation der vorliegenden Arbeit wies zu T1 insgesamt eine eher geringe Einschränkung bei Schmerz, Funktion und Lebensqualität auf. Möglicherweise führten diese geringen Einschränkungen dazu, dass kaum sichtbare Effekte erzielt werden konnten.

In diesem Kontext stellt sich die Frage, ob sich die verwendete Intervention für alle Patienten mit CURS eignet oder ob nach Subgruppen unterschieden werden sollte. Da Patienten mit Rückenschmerzen sehr unterschiedliche Ausprägungen bezüglich ihrer Symptomatik zeigen, ist es unter anderem gemäß der europäischen Leitlinie für das Management von CURS sinnvoll, den großen Anteil von Patienten mit unspezifischen Rückenschmerzen in Subgruppen einzuteilen, um geeignete Behandlungsprogramme zu finden [48]. Einige Studien untersuchten bereits spezifische Behandlungsverfahren für definierte Subgruppen und stellten bessere Ergebnisse dieser spezifischen Behandlung im Vergleich zu einer unspezifischen Therapie für die gesamte Patientengruppe fest $[49,50]$.

Zur statistischen Auswertung der vorliegenden Arbeit ist kritisch anzumerken, dass aus Gründen der Vergleichbarkeit und auf Basis vorliegender Literatur [30,42] zur Beurteilung der Ergebnisse die Mittelwerte verwendet wurden. Dies stellt bei auftretenden Ausreißern eine Gefahr der Verzerrung dar. Daher sollte zur transparenteren Darstellung der Ergebnisse künftig der Median zum Einsatz kommen.

Da die getroffenen Aussagen zur Durchführbarkeit des Graphästhesie-Trainings ausschließlich auf einer standardisierten Eingangsfrage gründen, sind sie mit Vorsicht zu betrachten.

\section{Stärken}

Die vorliegende Arbeit nutzte die bestmöglichen verfügbaren Messverfahren, die zahlreiche andere Publikationen bereits verwendeten [12, 21, 22, 41 -43]. Die eingesetzten Fragebögen sind reliabel und valide und damit für den Einsatz in dieser Pilotstudie geeignet. Außerdem erhob dieselbe Untersucherin alle Messungen vor und nach der Interventionsphase, sodass die Zuverlässigkeit der Messergebnisse hoch ist.

Alle Messverfahren wurden gemäß einer standardisierten Beschreibung und bei allen Probanden und zu allen Zeitpunkten nach einem eigens angefertigten Protokoll durchgeführt, um die Wahrscheinlichkeit von Messfehlern zu verringern. Das randomisierte, kontrollierte Modell mit Verblindung der Untersucherin stellt zudem ein grundsätzlich robustes Design dar.

\section{Schlussfolgerungen}

Diese Pilotstudie konnte keinerlei signifikante Unterschiede bezüglich der erhobenen Outcome-Parameter feststellen. Die Durchführbarkeit der Graphästhesie-Intervention wurde bestätigt, die Probanden berichteten von keinerlei Nebenwirkungen durch das Training. Folgestudien sollten die Durchführbarkeit jedoch mittels einer ausführlicheren quantitativen oder qualitativen Erhebung analysieren.

Die Autoren dieser Arbeit weisen explizit darauf hin, dass weitere Untersuchungen zu den Effekten des Graphästhesie-Trainings im Rahmen von multimodalen sensomotorischen Trainingsprogrammen bei Patienten mit CURS unter Einbezug einer Klassifikation in Subgruppen durchgeführt werden sollten. Insbesondere Letzteres verspricht aufgrund aktueller Forschungsergebnisse Therapieerfolge und kann die Versorgungssituation von Patienten mit CURS verbessern. Aus diesem Grund sollte die Klassifizierung in Subgruppen und die Zuordnung entsprechender Interventionen Gegenstand zukünftiger Forschungsarbeiten sein.

Folgestudien sollten die berechnete Stichprobengröße von $\mathrm{n}=54$ nicht unterschreiten.

Des Weiteren bedarf es weiterer Studien zur Ermittlung zufälliger und systematischer Messfehler bei sensomotorischen Testverfahren, um die gemessenen Unterschiede auf deren klinische Relevanz hin überprüfen zu können.

\section{Interessenkonflikt}

Die Autoren geben an, dass kein Interessenkonflikt besteht.

\section{Literatur}

[1] Schmidt CO, Raspe H, Pfingsten M et al. Back pain in the German adult population: prevalence, severity, and sociodemographic correlates in a multiregional survey. Spine (Phila Pa 1976) 2007; 32: 2005-2011

[2] Handelsblatt. Volkswirtschaftliche Schäden aufgrund chronischer Erkrankungen von Arbeitnehmern nach Krankheiten im Jahr 2010 (in Milliarden Euro). Handelsblatt 2012; 130: 16

[3] Barmer GEK. Heil- und Hilfsmittelreport. 2014 www.barmer.de/presse/ infothek/studien-und-reports/heil-und-hilfsmittelreport/report-201438588 (02.04.2018) 
[4] Deyo RA. Measuring the functional status of patients with low back pain. Arch Phys Med Rehabil 1988; 69: 1044-1053

[5] Airaksinen O, Brox Jl, Cedraschi C et al. Chapter 4. European guidelines for the management of chronic nonspecific low back pain. Eur Spine J 2006; 15 (Suppl. 2): S192-S300

[6] Bundesärztekammer (BÄK), Arbeitsgemeinschaft der Deutschen Ärztekammern, Kassenärztliche Bundesvereinigung KBV, Arbeitsgemeinschaft der Wissenschaftlichen Medizinischen Fachgesellschaften (AWMF) et al. Nationale VersorgungsLeitlinie Nicht-spezifischer Kreuzschmerz - Langfassung. 2017. Version 12. Aufl: www.leitlinien.de/mdb/ downloads/nvl/kreuzschmerz/kreuzschmerz-2aufl-vers1-lang.pdf (02.04.2018)

[7] Wand BM, O'Connell NE. Chronic non-specific low back pain - subgroups or a single mechanism? BMC Musculoskelet Disord 2008; 9: 11

[8] Van Tulder MW, Koes B, Malmivaara A. Outcome of non-invasive treatment modalities on back pain: an evidence-based review. Eur Spine J 2006; 15 (Suppl. 1): S64-S81

[9] Van Tulder MW, Assendelft W], Koes BW et al. Spinal radiographic findings and nonspecific low back pain. A systematic review of observational studies. Spine (Phila Pa 1976) 1997; 22: 427 -434

[10] Apkarian AV, Baliki MN, Geha PY. Towards a theory of chronic pain. Prog Neurobiol 2009; 87: 81-97

[11] Wand BM, Parkitny L, O'Connell NE et al. Cortical changes in chronic low back pain: current state of the art and implications for clinical practice. Man Ther 2011; 16: 15-20

[12] Luomajoki H, Moseley GL. Tactile acuity and lumbopelvic motor control in patients with back pain and healthy controls. Br J Sports Med 2011; 45: $437-440$

[13] Flor H, Braun C, Elbert T et al. Extensive reorganization of primary somatosensory cortex in chronic back pain patients. Neurosci Lett 1997; 224: $5-8$

[14] Lloyd D, Findlay G, Roberts N et al. Differences in low back pain behavior are reflected in the cerebral response to tactile stimulation of the lower back. Spine (Phila Pa 1976) 2008; 33: 1372-1377

[15] Maihofner C, Handwerker HO, Neundorfer B et al. Patterns of cortical reorganization in complex regional pain syndrome. Neurology 2003; 61: $1707-1715$

[16] Pleger B, Tegenthoff M, Ragert P et al. Sensorimotor retuning [corrected] in complex regional pain syndrome parallels pain reduction. Ann Neurol 2005; 57: 425-429

[17] Vartiainen NV, Kirveskari E, Forss N. Central processing of tactile and nociceptive stimuli in complex regional pain syndrome. Clin Neurophysiol 2008; 119: $2380-2388$

[18] Tracey I, Bushnell MC. How neuroimaging studies have challenged us to rethink: is chronic pain a disease? J Pain 2009; 10: 1113-1120

[19] Flor H, Elbert T, Knecht $\mathrm{S}$ et al. Phantom-limb pain as a perceptual correlate of cortical reorganization following arm amputation. Nature 1995; 375: $482-484$

[20] Moseley GL, Zalucki NM, Wiech K. Tactile discrimination, but not tactile stimulation alone, reduces chronic limb pain. Pain 2008; 137: 600-608

[21] Wand BM, Di Pietro F, George P et al. Tactile thresholds are preserved yet complex sensory function is impaired over the lumbar spine of chronic non-specific low back pain patients: a preliminary investigation. Physiotherapy 2010; 96: 317-323

[22] Moseley GL. I can't find it! Distorted body image and tactile dysfunction in patients with chronic back pain. Pain 2008; 140: 239-243

[23] Moberg E. Two-point discrimination test. A valuable part of hand surgical rehabilitation, e. g. in tetraplegia. Scand J Rehabil Med 1990; 22: $127-134$

[24] Amshoff T, von Aufschnaiter D, Bader-Johansson C et al. physiolexikon: Physiotherapie von A bis Z. Stuttgart: Thieme; 2010
[25] Knecht S, Kunesch E, Schnitzler A. Parallel and serial processing of haptic information in man: effects of parietal lesions on sensorimotor hand function. Neuropsychologia 1996; 34: 669-687

[26] Staal JB. HE, Heijmanns M, Kiers H et al. KNGF Guideline Low back Pain. 2013 www.ipts.org.il/_Uploads/dbsAttachedFiles/ low_back_pain_practice_guidelines_2013.pdf (02.04.2018)

[27] Waddell G. 1987 Volvo award in clinical sciences. A new clinical model for the treatment of low-back pain. Spine (Phila Pa 1976) 1987; 12: 632-644

[28] Waddell G. An approach to backache. Br J Hosp Med 1982; 28: 190 191, 193-194, passim

[29] Chapman JR, Norvell DC, Hermsmeyer JT et al. Evaluating common outcomes for measuring treatment success for chronic low back pain. Spine (Phila Pa 1976) 2011; 36: S54-S68

[30] Morfeld M, Kirchberger I, Bullinger M. SF-36 Fragebogen zum Gesundheitszustand - deutsche Version des Short-Form-36 Health Survey. Göttingen: Hogrefe; 2011

[31] Ellert U, Kurth BM, Robert Koch-Institut Berlin.. Methodische Betrachtungen zu den Summenscores des SF-36 anhand der erwachsenen bundesdeutschen Bevölkerung. Bundesgesundheitsbl-Gesundheitsforsch Gesundheitsschutz. 2004; 47: 1027-1032

[32] Bullinger M. German translation and psychometric testing of the SF-36 Health Survey: preliminary results from the IQOLA Project. International Quality of Life Assessment. Soc Sci Med 1995; 41: 1359-1366

[33] Roland M, Fairbank J. The Roland-Morris Disability Questionnaire and the Oswestry Disability Questionnaire. Spine (Phila Pa 1976) 2000; 25: $3115-3124$

[34] Junge A, Mannion AF. Questionnaires for patients with back pain. Diagnosis and outcome assessment. Orthopade 2004; 33: 545- 552

[35] Roland M, Morris R. A study of the natural history of back pain. Part I: development of a reliable and sensitive measure of disability in low-back pain. Spine (Phila Pa 1976) 1983; 8: 141-144

[36] Wiesinger GF, Nuhr M, Quittan M et al. Cross-cultural adaptation of the Roland-Morris questionnaire for German-speaking patients with low back pain. Spine (Phila Pa 1976) 1999; 24: 1099-1103

[37] Radbruch L, Loick G, Kiencke P et al. Validation of the German version of the Brief Pain Inventory. J Pain Symptom Manage 1999; 18: 180-187

[38] Cleeland CS. The Brief Pain Inventory - User Guide. 2009 www.mdanderson.org/documents/Departments-and-Divisions/Symptom-Research/BPI_UserGuide.pdf (02.04.2018)

[39] Cleeland CS, Ryan KM. Pain assessment: global use of the Brief Pain Inventory. Ann Acad Med Singap 1994; 23: 129-138

[40] Faul F, Erdfelder E, Lang AG et al. G*Power 3: A flexible statistical power analysis program for the social, behavioral, and biomedical sciences. 2007 www.gpower.hhu.de/fileadmin/redaktion/Fakultaeten/Mathematisch-Naturwissenschaftliche_Fakultaet/Psychologie/AAP/gpower/GPower3-BRM-Paper.pdf (02.04.2018)

[41] Wand BM, O'Connell NE, Di Pietro F et al. Managing chronic nonspecific low back pain with a sensorimotor retraining approach: exploratory multiple-baseline study of 3 participants. Phys Ther 2011; 91: 535-546

[42] Wälti P, Kool J, Luomajoki H. Short-term effect on pain and function of neurophysiological education and sensorimotor retraining compared to usual physiotherapy in patients with chronic or recurrent non-specific low back pain, a pilot randomized controlled trial. BMC Musculoskelet Disord 2015; 16: 83

[43] Ryan C, Harland N, Drew BT et al. Tactile acuity training for patients with chronic low back pain: a pilot randomised controlled trial. BMC Musculoskelet Disord 2014; 15: 59

[44] Ehrenbrusthoff K, Ryan CG, Grüneberg C et al. The intra- and inter-observer reliability of a novel protocol for two-point discrimination in individuals with chronic low back pain. Physiological Measurement 2016; 37: $1074-1088$ 
[45] Catley M], O'Connell NE, Berryman C et al. Is tactile acuity altered in people with chronic pain? A systematic review and meta-analysis. J Pain 2014; 15: $985-1000$

[46] Catley MJ, Tabor A, Wand BM et al. Assessing tactile acuity in rheumatology and musculoskeletal medicine-how reliable are two-point discrimination tests at the neck, hand, back and foot? Rheumatol (Oxford) 2013; 52: $1454-1461$

[47] Wand BM, Catley MJ, Luomajoki HA et al. Lumbar tactile acuity is near identical between sides in healthy pain-free participants. Man Ther 2014; 19: $504-507$
[48] Airaksinen O, Brox JI, Cedraschi C et al. Chapter 4. European guidelines for the management of chronic nonspecific low back pain. Eur Spine J 2006; 15 (Suppl. 2): S192-S300

[49] Fersum KV, Dankaerts W, O’Sullivan PB et al. Integration of subclassification strategies in randomised controlled clinical trials evaluating manual therapy treatment and exercise therapy for non-specific chronic low back pain: a systematic review. Br J Sports Med 2010; 44: 1054-1062

[50] Billis EV, McCarthy C], Oldham JA. Subclassification of low back pain: a cross-country comparison. Eur Spine J 2007; 16: 865-879 